\title{
AN ASYMPTOTIC-INDUCED SCHEME FOR NONSTATIONARY TRANSPORT EQUATIONS IN THE DIFFUSIVE LIMIT
}

\author{
$A X E L K L A R^{*}$
}

Abstract. An asymptotic-induced scheme for nonstationary transport equations with the diffusion scaling is developed. The scheme works uniformly for all ranges of nean free paths. It is based on the asymptotic analysis of the diffusion limit of the transport equation.

A theoretical investigation of the behaviour of the scheme in the diffusion limit is given and an approximation property is proven. Moreover, mumerical results for different physical situations are shown and the unifom convergence of the scheme is established numerically.

Key words. transport equations, asymptotic analysis, diffusion limit, numerical methods for stiff equations

AMS subject classifications. $82070,05 \mathrm{M} 00,35 \mathrm{~B} 25$

1. Introduction. Transport equations are used to describe many physical phenomena. Some of the best known examples are neutron transport, radiative transfer equations, semiconductors or gas kinetics. The situation for small mean free paths is mathematically described by an asymptotic analysis. Depending on the transport equation and on the kind of scaling, different limit equations are obtained. For example the gas kinetic equations may lead to Euler or (in)compressible Navier Stokes equations. The limit equation for small mean free paths of radiative transfer, neutron transport, or semiconductor equations is the diffusion and the drift-diffusion equation, respectively. We refer to $[3,4,12,18,20,28]$ and $[2,6,8,10]$.

The main problem for numerical work on transport equations in these regimes is the stiffness of the equations for small mean free paths. For standard numerical schemes one has to use a very fine and expensive discretization with a discretization size depending on the mean free path. Moreover, in general a full resolution of the relaxation process is not necessary. The general aim is to develop numerical schemes working uniformly for different regimes. In particular, the discretization size should be independent of the mean free path. In recent years there has been a lot of work on numerical methods for kinetic equations in stiff regimes. For example, stationary transport equations in the diffusion limit have been considered, e.g., in $[14,15,22,21]$. Nonstationary kinetic equations with a scaling leading to first order hydrodynamic equations like the Euler equation are treated in $[7,9]$. Usually for the latter case a fractional step method with a semi-implicit treatment of the equations is used. For general work on implicit methods for transport equations we refor to [27] and refor ences therein. We mention here also work on implicit methods for the full Boltzmann equation, see [5]. Moreover; the relaxation limit of transport equations may be used to develop schemes for the hydrodynamic equations themselves. These schemes have been developed by many authors. For a recent general approach to these so called relaxed or kinetic schemes we refer to [16].

The present work considers a scheme for nonstationary transport equations with a scaling leading to the diffusion equation as the limit equation. The different space time scalings involved in the problem are treated in a proper way. We use the standard perturbation procedure leading from the transport to the diffusion equation.

*FB Nathematk, University of Kaiserslauterm, 67663 Kaserslautern, Germany, (klar@mathematik uni-k1, de). 
Essentially the problem is transformed into a system of equations of relaxation form and then a fractional step method is used. The analysis of the resulting problem is based on ideas developed in [7]. Including the results of a boundary layer analysis in the scheme, kinetic boundary layers are also treated in a correct way. Sections 2 and 3 contain a description of the results of the standard asymptotic procedure and the presentation of the time discretization in our scheme. In Section 4 the diffusion limit of the scheme is considered. In Section 5 the fully discretized equations are presented. An approximation property for different ranges of the mean free path is proven in 6 . Section 7 contains numerical results for several examples and a numerical comparison with other schemes.

Finishing the introduction we mention that the ideas developed in this paper can be transfered to the gas kinetic and the semiconductor case, where the above scaling leads in the limit to the incompressible Navier-Stokes equation and the drift-diffusion equation respectively. In particular, in the gas dynamic case a more careful use has to be made of the perturbation procedure leading from the Boltzmann equation to the incompressible Navier-Stokes equation. This problem will be treated in a separate paper.

2. The Equations. We consider transport equations of the following form

$$
\partial_{t} f+v \cdot \nabla_{x} f=Q(f)+G(x)
$$

where $f=f(x, v, t)$ with $x \in \Omega \subset \mathbb{R}^{d}, d=1,2,3, v=\left(v_{1}, v_{2}, v_{3}\right) \in S \subseteq \mathbb{R}^{3}, t \in[0, \infty)$, where $S$ is assumed to be the unit sphere around 0 in $\mathbb{R}^{3}$. The collision operator $Q$ is defined by

$$
Q f(x, v, t)=\sigma(x)(K f-f)(x, v, t)
$$

with $0<\sigma_{1}<\sigma(x)<\sigma_{2}$, where $\sigma_{1}, \sigma_{2}$ are some constants and the scattering cross section $\sigma$ is independent of $v . K$ is an integral operator

$$
K f(x, v, t)=\int_{S} s\left(v, v^{\prime}\right) f\left(x, v^{\prime}, t\right) d v^{\prime}
$$

$s$ symmetric in $v$ and $v^{\prime}$, rotationally invariant, $0<s_{1}<s\left(v, v^{\prime}\right)<s_{2}$, where $s_{1}$, s $s_{2}$ are some constants, and $\int_{S} s\left(v, v^{\prime}\right) d v^{\prime}=1 . K$ is compact. The collision operator $K-1$. has as collision invariants only constants and is negative in a suitable function space. The source term $G(x) \geq 0$ is assumed to be independent of $v$. Initial and boundary conditions are given by

$$
f(x, v, 0)=g(x, v) \geq 0, x \in \Omega, v \in S
$$

and

$$
f(x, v, t)=k(x, v, t) \geq 0, x \in \partial \Omega, v \cdot n<0,
$$

where $\partial D$ is the boundary of $\Omega$ and $n=n(x)$ the outer normal of $\partial \Omega$ at the point $x$. See [3] for a thorough theoretical investigation of this equation. Extensions of the following to other cases like, e.g., $v$-dependence of $\sigma$ and $G$ are possible.

Introducing the usual diffusion space-time scaling $x \rightarrow \frac{x}{\epsilon}$ and $t \rightarrow \frac{t}{\epsilon^{2}}$, where $\epsilon$ is the mean free path and scaling $G(x) \rightarrow \epsilon^{2} G(x)$, one obtains the scaled equations

$$
\partial_{t} f+\frac{1}{\epsilon} v \cdot \nabla_{x} f=\frac{1}{\epsilon^{2}} \sigma(K-I)(f)+G(x) .
$$


With the standard perturbation procedure, see e.g. $[3,4,12,20]$ the limit equation for (2.2) as $\epsilon$ tends to 0 can be derived by writing $f$ as

$$
f=f_{0}+\epsilon f_{1}+\cdots
$$

One obtains

$$
f_{0}=\Theta(x, t)
$$

and up to a constant

$$
f_{1}=Q^{-1}\left(v \cdot \nabla_{x} f_{0}\right)=Q^{-1}(v) \cdot \nabla_{x} f_{0}
$$

Let $h=\left(h_{1}, h_{2}, h_{3}\right)$ be the solution of $(I-K)\left(h_{i}\right)=v_{i}$ with $\int_{S} h_{i}(v) d v=0$ for $i \in 1,2,3$. Since by assumption $s$ is rotationally invariant, it follows [3] that $\forall i, j \in$ $1,2,3: \frac{1}{5 \mid} \int_{S} v_{i} h_{j} d v=D \delta_{i, j}$. Then $\Theta(x, l)$ is the solution of the diffusion equation

$$
\partial_{t} \Theta(x, t)-D \nabla_{x} \cdot\left(\frac{\nabla_{x} \Theta}{\sigma}\right)=G(x)
$$

Doing a boundary layer analysis, one observes that the correct zeroth order boundary conditions for the diffusion equation are given by a kinetic half space problem: Let $\chi^{2}(y, v, t)$ be the bounded solution of the following halfspace problem at $x$

$$
\begin{array}{r}
v n(x) \partial_{y} \chi^{x}=k \chi^{2}-\chi^{x}, y \in \mathbb{R}^{-} \\
\chi^{x}(0, v, t)=k(x, v, t), x \in \partial \Omega, v \cdot n<0
\end{array}
$$

Then

$$
\Theta(x, t)=\chi^{x}(-\infty, t), x \in \partial \Omega
$$

Here $\chi^{n}(-\infty, t)$ is independent of $v$.

Remark: In the absorbing case the scaled equation (2.2) is changed into

$$
\partial_{t} f+\frac{1}{\epsilon} v \cdot \nabla_{x} f=\frac{\sigma}{\epsilon^{2}} K(f)-\left(\frac{\sigma}{\epsilon^{2}}+\sigma_{A}\right) f+G(x),
$$

where $\sigma_{A}$ is the absorption cross section. The diffusion equation turns into

$$
\partial_{t} \Theta(x, t)-D \nabla_{x} \cdot\left(\frac{\nabla_{x} \Theta}{\sigma}\right)+\sigma_{A} \Theta=G(x)
$$

3. The Numerical Scheme. For a numerical scheme for the transport equation in the small mean free path limit it is desirable that varying mean free paths $\epsilon$ can be treated with a fixed discretization such that it is not necessary to adapt the time step once the mean free paths tend to 0. Moreover, it is also desirable that the scheme is in the limit $\epsilon \rightarrow 0$ a good discretization of the diffusion equation.

These points are obviously not fullfilled for a simple explicit time discretization of (2.2) like

$$
f^{k+1}=f^{k}+\Delta t\left[-\frac{v}{\epsilon} \nabla x f^{k}+\frac{1}{e^{2}} \sigma(K-I)\left(f^{k}\right)+G(x)\right]
$$

since, as $\epsilon$ tends to 0 , the time step must be shrinked due to stability considerations in order to treat the advection term (the CFL condition has to be fulfilled) and the 
collision term properly. Therefore, large computation times are needed for small mean free path for such a scheme. In contrast, for a fully implicit discretization

$$
f^{k+1}=f^{k}+\Delta t\left[-\frac{v}{\epsilon} \nabla_{x} f^{k+1}+\frac{1}{\epsilon^{2}} \sigma(K-I)\left(f^{k+1}\right)+G(x)\right]
$$

there is no restriction on the time step due to stability considerations. However, one has to solve a stationary equation in every time step, which is again time consuming. We montion that, due to the development of fast multigrid algorithms $[19,24,25,26]$, for the stationary equation, computation times for a fully implicit scheme are strongly reduced. A numerical comparison of these types of algorithms with the one devcloped here is presented in Section 7 .

The aim in this work is to develop a semi-implicit scheme treating only such terms in an implicit way for which it is necessary to do so in order to obtain a scheme working uniformly in $\epsilon$. In particular, due to the different advection $\left(\frac{1}{\epsilon}\right)$ and scattering $\left(\frac{1}{\epsilon^{2}}\right)$ scales, it is in the original formulation (2.2) not clear whether the advection has to be treated implicitely or not. One may nevertheless discretize the original equations in a straightforward way by treating the advection explicitely and the scattering term in an implicit way:

$$
f^{k+1}=f^{k}+\Delta t\left[-\frac{v}{\epsilon} \nabla_{x} f^{k}+\frac{1}{\epsilon^{2}} \sigma(K-I)\left(f^{k+1}\right)+G(x)\right] .
$$

This simple type of discretization has several drawbacks compared to the scheme developed below, we discuss them at the end of Section 4 .

We suggest to use the standard perturbation procedure to transform equation (2.2) into two equations. A fractional step scheme with a semi-implicit procedure is then used for the resulting equations. The idea is to follow the expansion procedure, write $f$ as $f=f_{0}+\epsilon f_{1}$ and collect suitable terms together, such that only terms on the scale $\frac{1}{\epsilon^{2}}$ are involved.

Let $\left(f_{0}, f_{1}\right)$ be the solution of the set of equations

$$
\begin{aligned}
\partial_{t} f_{0}+v \cdot \nabla_{x} f_{1} & =\frac{1}{\epsilon^{2}} \sigma\left(K f_{0}-f_{0}\right)+G(x) \\
\partial_{t} f_{1} & =\frac{1}{\epsilon^{2}}\left(-v \cdot \nabla_{x} f_{0}+\sigma\left(K f_{1}-f_{1}\right)\right)
\end{aligned}
$$

We take the inital and boundary values

$$
f_{0}(x, v, 0)+\epsilon f_{1}(x, v, 0)=g(x, v), x \in \Omega, v \in S
$$

and

$$
\begin{aligned}
& f_{0}(x, v, t)+\epsilon f_{1}(x, v, t)=k(x, v, t), x \in \partial \Omega, v \cdot n<0 \\
& f_{0}(x, v, t)-\epsilon f_{1}(x, v, t)=q(x, v, t), x \in \partial \Omega, v \cdot n>0 .
\end{aligned}
$$

One observes that $f_{0}+\epsilon f_{1}$ fulfills the original equation $(2.2)$ and the initial and boundary conditions. It is therefore the desired solution of the original problem.

The results of the boundary layer analysis, see, e.g. [3], are included in the scheme by choosing $h$ in the following way:

Let $\chi^{x}(y, v, t)$ be the solution of the halfspace problem (2.3). Since the outgoing 
function at the boundary for the kinetic problem (2.2) is the same as the outgoing solution of the half space problem for $\epsilon$ tending to 0 , we define

$$
q(x, v, t)=\chi^{x}(0, v, t), x \in \partial \Omega, v \cdot n>0 .
$$

In the limit $\epsilon$ tending to 0 we obtain in this way the correct boundary value. For $k=k(x, t)$ independent of $v$ we get $q(x, v, t)-k(x, t), v \cdot n>0$. It is obviously not reasonable to determine the outgoing function by solving the halfspace problem. This would need too much computing time. Here a fast approximate scheme as in [11] or [17] is needed to determine the outgoing function. For example a first approximation is given by choosing simply an approximation $\tilde{\chi}^{x}(-\infty, t)$ of the asymptotic value $\chi^{x}(-\infty, t)$ of the halfspace problem as the outgoing function:

$$
q(x, v, t)=\chi^{x}(-\infty, t), x \in \partial \Omega, v, n>0 .
$$

The simplest approximation of $\chi^{n}(-\infty, t)$ is given by equalizing the half range fluxes of the halfspace problem at 0 and $\infty$ :

$$
\chi^{x}(-\infty, t)=\frac{\int_{v, n \leq 0} v \cdot n k(x, v, t) d v}{\int_{v \cdot n<0} v \cdot n d v}
$$

A more sophisticated approximation for $q$, see [17], is given by

$$
\begin{aligned}
& x^{n}(-\infty, t)=\frac{\int_{v n<0} v \cdot n k(x, v, t) d v}{\int_{v n<0} v \cdot n d v}+ \\
& \frac{1}{D} \frac{1}{4 \pi} \int_{v \cdot n<0}(v \cdot n)^{2}\left[k(x, v, t)-\frac{\int_{v \cdot n<0^{2}} v n k(x, v, t) d v}{\int_{v \cdot n<0} v \cdot n d v}\right] d n
\end{aligned}
$$

and

$$
\begin{aligned}
q(x, v, t)= & \tilde{\chi}^{x}(-\infty, t)+\frac{1}{4 \pi} \int_{w \cdot n<0} \frac{w \cdot n}{(w-v) \cdot n}\left[s(v, w)-\frac{(w \cdot n)^{2}}{L}\right] \\
& {\left[k(x, w, t)-\tilde{\chi}^{x}(-\infty, t)\right] d w, x \in \partial \Omega, v \cdot n>0 . }
\end{aligned}
$$

We remark that a correct treatment of the boundary conditions is important, in particular, if zeroth order kinetic boundary layers are present and one is using a coarse spatial grid not resolving the layer. See Section 7 for some examples. Using the approximations above one obtains a good approximation of the solution with a first: order boundary layer even if only a very coarse grid is used. The first approximation yields in general already very good results as can be seen in the numerical experiments. in Section 7. However, in certain situations the use of the second approximation might be necessary to obtain an improved accuracy, compare Figure 7.6 in Section 7.

The system of equations $(3.4,3.5)$ will be solved with a fractional step scheme:

Step 1:

$$
\begin{aligned}
\partial_{t} f_{0}+v \cdot \nabla_{x} f_{1} & =G(x) \\
\partial_{t} f_{1} & =0
\end{aligned}
$$

Step 0:

$$
\begin{aligned}
& \partial_{t} f_{0}=\frac{1}{\epsilon^{2}} \sigma\left(K f_{0}-f_{0}\right) \\
& \partial_{t} f_{1}=\frac{1}{\epsilon^{2}}\left(-v \cdot \nabla_{x} f_{0}+\sigma\left(K f_{1}-f_{1}\right)\right)
\end{aligned}
$$


For Step 1 an explicit discretization will be used, Step 2 is discretized implicitely to treat the stiffness of the equations in a correct way.

Let $\Delta t$ denote the time step and $f_{0}^{k}, f_{1}^{k}, k=0, \cdots, n=\frac{t}{\Delta t}$ the time iterations approximating $f_{0}(x, v, k \Delta t), f_{1}(x, v, k \Delta t)$. The initial and boundary values are given as above. Introducing the notation

$$
<->=\frac{1}{|S|} \int_{S} d v
$$

the time discretization is then given by the following:

Step 1:

$$
\begin{array}{r}
f_{0}^{k+\frac{1}{2}}=f_{0}^{k}-\Delta t v \cdot \nabla_{x} f_{1}^{k}+G \Delta t \\
f_{1}^{k+\frac{1}{2}}=f_{1}^{k}
\end{array}
$$

Step 2:

$$
\begin{aligned}
& f_{0}^{k+1}=f_{0}^{k+\frac{1}{2}}+\frac{\Delta t}{\epsilon^{2}} \sigma\left(K f_{0}^{k+1}-<f_{0}^{k+1}>+<f_{0}^{k+\frac{1}{2}}>-f_{0}^{k+1}\right) \\
& f_{1}^{k+1}=f_{1}^{k+\frac{1}{2}}+ \\
& \frac{\Delta t}{\epsilon^{2}}\left[-v \cdot \nabla_{x} f_{0}^{k+1}+\sigma\left(K f_{1}^{k+1}-<f_{1}^{k+1}>+<f_{1}^{k+\frac{1}{2}}>-f_{1}^{k+1}\right)\right]
\end{aligned}
$$

Rewriting $(3.13,3.14)$ we obtain

$$
\begin{aligned}
& \quad\left(1+\frac{\Delta t}{\epsilon^{2}} \sigma\right) f_{0}^{k+1}-\frac{\Delta t}{\epsilon^{2}} \sigma\left(K f_{0}^{k+1}-<f_{0}^{k+1}>\right) \\
& =f_{0}^{k+\frac{1}{2}}+\frac{\Delta t}{\epsilon^{2}} \sigma<f_{0}^{k+\frac{1}{2}}>
\end{aligned}
$$

and

$$
\begin{aligned}
& \left(1+\frac{\Delta t}{\epsilon^{2}} \sigma\right) f_{1}^{k+1}-\frac{\Delta t}{\epsilon^{2}} \sigma\left(K f_{1}^{k+1}-<f_{1}^{k+1}>\right) \\
= & f_{1}^{k+\frac{1}{2}}+\frac{\Delta t}{\epsilon^{2}} \sigma<f_{1}^{k+\frac{1}{2}}>-\frac{\Delta t}{\epsilon^{2}} v \cdot \nabla_{x} f_{0}^{k+1}
\end{aligned}
$$

This leads to

Step 2:

$$
\begin{aligned}
& f_{0}^{k+1}=A f_{0}^{k+\frac{1}{2}}+B<f_{0}^{k+\frac{1}{2}}> \\
& f_{1}^{k+1}=A f_{1}^{k+\frac{1}{2}}+B\left[<f_{1}^{k+\frac{1}{2}}>-\frac{v}{\sigma} \cdot \nabla_{x} f_{0}^{k+1}\right],
\end{aligned}
$$

where the operator $A$ is defined by

$$
A=\left(I+\frac{\Delta t}{\epsilon^{2}} \sigma(I-K+<>)\right)^{-1}=\frac{\epsilon^{2}}{\Delta t \sigma}\left(\frac{\epsilon^{2}}{\Delta t \sigma} I+(I-K+<>)\right)^{-1}
$$

and

$$
B=\frac{\Delta t}{\epsilon^{2}} \sigma A=\left(\frac{\epsilon^{2}}{\Delta t \sigma} I+(I-K+<>)\right)^{-1}
$$


Here $I$ denotes the identity. The operator

$$
\left(\frac{\epsilon^{2}}{\Delta t \sigma} I+(I-K+<>)\right)
$$

is positive and invertible with a bounded inverse in a suitable function space for all $\epsilon \geq 0, \Delta t>0$, since $K$ is compact and $I-K$ is positive having only the constants as collision invariants.

For example in the case of one-group transport with $K-<<$ we obtain

$$
A=\left(1+\frac{\Delta t}{t^{2}} \sigma\right)^{-1} T
$$

and

$$
B=\left(\frac{\epsilon^{2}}{\Delta t_{0}}+1\right)^{-1} I
$$

In this case the semi-implicit scheme reduces to a fully explicit one In general, in each time step we have to solve in Step 2 two linear Fredholm integral equation of the form

$$
\left(\frac{\epsilon^{2}}{\Delta t 0} I+(I-K+<>)\right) f=g
$$

This may be achieved by standard methods $[1,13]$.

Remark: In the absorbing case one proceeds as described above treating the absorption in an explicit way. Then one obtains

Step 1:

$$
\begin{aligned}
& \int_{0}^{k+\frac{1}{2}}=\int_{0}^{k}-\Delta t\left(v \cdot \nabla_{x} \int_{1}^{k}+\sigma_{A} f_{0}^{k}\right)+G \Delta t \\
& f_{1}^{k+\frac{1}{2}}=f_{1}^{k}-\Delta t \sigma_{A} f_{1}^{k}
\end{aligned}
$$

Step 2 is unchanged.

4. The Diffusion Limit. We start with the investigation of the behaviour of the time discretized scheme as $\epsilon$ tends to 0 for fixed $\Delta t$.

As $\epsilon \rightarrow 0$ the operators $A$ and $B$ have the following behaviour: Introducing suitable spaces, e.g. $L^{2}(S)$, we have for $\Delta t$ fixed and $\epsilon$ small, since $(I-K+<>)$ is positive the following:

$$
|A| \leq O\left(\frac{e^{2}}{\Delta t \sigma}\right)
$$

and

$$
\left|B-(I-K+<)^{1}\right| \leq O\left(\frac{e^{2}}{\Delta t \sigma}\right)
$$

Using these stimates we get that the scheme reduces in the diffusion limit, 6 tending to 0 , to the following

Step 1 :

$$
\begin{array}{r}
f_{0}^{k+\frac{1}{2}}=f_{0}^{k}-\Delta t v \cdot \nabla_{x} f_{1}^{k}+G \Delta t \\
f_{1}^{k+\frac{1}{2}}=f_{1}^{k}
\end{array}
$$


Step 2:

$$
\begin{aligned}
& f_{0}^{k+1}=(I-K+<>)^{-1}<f_{0}^{k+\frac{1}{2}}> \\
& f_{1}^{k+1}=(I-K+<>)^{-1}\left[<f_{1}^{k+\frac{1}{2}}>-\frac{v}{\sigma} \cdot \nabla_{x} f_{0}^{k+1}\right]
\end{aligned}
$$

Morcover, we have

$$
(I-K+<>)<f>=<f>
$$

and

$$
(I-K+<>) h(v)=v
$$

where $h$ was defined in Section 2. This yields

Step 2:

$$
\begin{aligned}
& f_{0}^{k+1}=<f_{0}^{k+\frac{1}{2}}> \\
& f_{1}^{k+1}=f_{1}^{k+\frac{1}{2}}>-\frac{h(v)}{\sigma} \nabla_{x}<f_{0}^{k+\frac{1}{2}}>
\end{aligned}
$$

Considering Step 2 and Step 1 together we obtain for $\epsilon=0$

$$
\begin{array}{r}
f_{0}^{k+1}=<f_{0}^{k+1}>=<f_{0}^{k+\frac{1}{2}}>=<f_{0}^{k}>-\Delta t \nabla_{x} \cdot<v f_{1}^{k}>+G \Delta t \\
=<f_{0}^{k-\frac{1}{2}}>+\Delta t D \nabla_{x} \cdot\left(\frac{\nabla_{x}<f_{0}^{k-\frac{1}{2}}>}{\sigma}\right)+G \Delta t \\
=<f_{0}^{k}>+\Delta t D \nabla_{x} \cdot\left(\frac{\nabla_{x}<f_{0}^{k}>}{\sigma}\right)+G \Delta t
\end{array}
$$

or

$$
\Theta^{k+1}=\Theta^{k}+\Delta t D \nabla_{x} \cdot\left(\frac{\nabla_{x} \Theta^{k}}{\sigma}\right)+G \Delta t
$$

This is the simplest explicit time discretization for the diffusion equation. The boundary conditions for the diffusion equation that are given in the limit by the solution of the halfspace problem (2.3) fit to the boundary conditions for the kinetic scheme as defined in the last section.

We finish this section by comparing the above scheme with the scheme $(3.3)$ in Section 3. Doing the standard asymptotic analysis [21] we get for (3.3) as $\epsilon \rightarrow 0$

$$
<f_{0}^{k+1}>=<f_{0}^{k}>+\Delta t D \nabla_{x} \cdot\left(\frac{\nabla_{x}<f_{0}^{k-1}>}{\sigma}\right)+G(x) .
$$

This means we obtain an explicit discretization of the diffusion equation as for the above scheme, but due to the $\left\langle f_{0}^{k-1}\right\rangle$ term, it is not the usual one. This type of discretization of the diffusion equation is worse in terms of accuracy and stability than (4.4). For example, doing a stability analysis one observes that only time steps are allowed which are half the size of those that can be used in (4.4). This is essentially due to the fully explicit treatment of the advection term in (3.3). Moreover, the scheme developed in Section 3 gives the possibility to treat for example the collision terms in a semi-implicit way as given in $(3.13,3.14)$. This is at least for one-group transport with $K=<>$ a decisive advantage, since the semi-implicit scheme presented here reduces in this case to a fully explicit one. If one would be trying to do the same thing based on the scheme (3.3) it would turn out that the limit equation is not any more the diffusion equation. 
5. The Fully Discretized Equations. We restrict from now on for notational simplicity to the case, where $f_{0}$ and $f_{1}$ depend only on the first space coordinate: The domain under consideration is $[0, L]$. Moreover, we consider only the space discretization. The velocity space can be treated by using standard discretizations, see, e.g., [23].

We define a staggered grid $x_{i}-i \Delta x, i-0, \cdots, N$ with $N-\frac{L}{\Delta x}$, and $x_{i-\frac{1}{2}}=$ $\left(i-\frac{1}{2}\right) \Delta x, i=0, \cdots, N+1$.

We use the notation

$$
\begin{aligned}
& f_{0}^{k}=f_{0}^{k}(i) \sim f_{0}(i \Delta x, v, k \Delta t), \\
& f_{1}^{k}=f_{1}^{k}(i) \sim f_{1}\left(\left(i-\frac{1}{2}\right) \Delta x, v, k \Delta t\right)
\end{aligned}
$$

and

$$
\sigma=\sigma(i) \sim \sigma\left(\left(i-\frac{1}{2}\right) \Delta x\right)
$$

Moreover the operators $D^{+}$and $D$ are defined as

$$
\begin{aligned}
& D^{+} f(i)=f(i+1)-f(i) \\
& D-f(i)=f(i)-f(i-1) .
\end{aligned}
$$

The discretization of the initial values is straightforward. The boundary conditions are discretized by

$$
\begin{array}{r}
f_{0}^{k}(0)+\frac{\epsilon}{2}\left(f_{1}^{k}(0)+f_{1}^{k}(1)\right)=k(0, v, t), v_{1}>0 \\
f_{0}^{k}(0)-\frac{\epsilon}{2}\left(f_{1}^{k}(0)+f_{1}^{k}(1)\right)=q(0, v, t), v_{1}<0 \\
f_{0}^{k}(N)+\frac{\epsilon}{2}\left(f_{1}^{k}(N)+f_{1}^{k}(N+1)\right)-k(L, v, t), v_{1}<0 \\
f_{0}^{k}(N)-\frac{\epsilon}{2}\left(f_{1}^{k}(N)+f_{1}^{k}(N+1)\right)=q(L, v, t), v_{1}>0 .
\end{array}
$$

Discretizing the $f_{0}$ derivative in (3.16) with $D$ and the $f_{1}$ derivative in (3.11) with $D_{+}$yields the following scheme

Step 1:

$$
\begin{array}{r}
f_{0}^{k+\frac{1}{2}}=f_{0}^{k}-v_{1} \Delta t \frac{D^{+}}{\Delta x} f_{1}^{k}+G \Delta t \\
f_{1}^{k+\frac{1}{2}}=f_{1}^{k}
\end{array}
$$

Step 2:

$$
\begin{aligned}
& f_{0}^{k+1}=A f_{0}^{k+\frac{1}{2}}+B<f_{0}^{k+\frac{1}{2}}> \\
& f_{1}^{k+1}=A f_{1}^{k+\frac{1}{2}}+B\left[<f_{1}^{k+\frac{1}{2}}>-\frac{v_{1}}{\sigma \cdot} \frac{D}{\Delta x} f_{0}^{k+1}\right]
\end{aligned}
$$


In the limit for small $\epsilon$ we obtain the space discretized diffusion equation

$$
\begin{array}{r}
f_{0}^{k+1}=<f_{0}^{k+1}>-<f_{0}^{k+\frac{1}{2}}>=<f_{0}^{k}>-\Delta t \frac{D^{+}}{\Delta x}<v_{1} f_{1}^{k}>+G \Delta t \\
=<f_{0}^{k-\frac{1}{2}}>+\frac{\Delta t}{(\Delta x)^{2}} D D^{+}\left(\frac{D-<f_{0}^{k-\frac{1}{2}}>}{\sigma}\right)+G \Delta t \\
=<f_{0}^{k}>+\frac{\Delta t}{(\Delta x)^{2}} D D^{+}\left(\frac{D_{-}<f_{0}^{k}>}{\sigma}\right)+G \Delta l
\end{array}
$$

or

$$
\Theta^{k+1}=\Theta^{k}+\frac{\Delta t}{(\Delta x)^{2}} D D^{+}\left(\frac{D-\Theta^{k}}{\sigma}\right)+G \Delta t
$$

This is a standard explicit discretization of the diffusion equation. In particular, we obtain independent of the size of the discretization $\Delta x$ a good discretization of the limit equation for all ranges of the mean free path. The discretization possesses all diffusion limits, the so called thin, intermediate and thick diffusion limit, see [22]. We observe, that we need in the limit a relation like

$$
\Delta t \leq \frac{(\Delta x)^{2}}{2} \frac{\sigma}{D}
$$

as for the diffusion equation, to obtain positivity and stability of our scheme. This condition may be relaxed for $\epsilon$ large.

6. A Uniform Approximation Property. In this section we prove a uniform approximation property of our scheme. We give an estimate for the consistency error, considering the integral form of equations $(3.4,3.5)$ assuming that the true solution is smooth.

Written in integral form the equations for $f_{0}(t)$ and $f_{1}(t)$ are for the Cauchy problem and one space dimension

$$
\begin{aligned}
& f_{0}(t)=e^{-\frac{\mathrm{t \sigma}}{e^{2}}} g(x)+\int_{0}^{t} e^{-\frac{(t-s) \cdot \sigma}{\epsilon^{2}}}\left[\frac{\sigma K f_{0}(s)}{\epsilon^{2}}-v_{1} \partial_{x} f_{1}(s)+G\right] d s \\
& f_{\mathrm{I}}(t)=\int_{0}^{t} e^{-\frac{(t-s) \sigma}{\epsilon^{2}}}\left[\frac{\sigma K f_{1}(s)}{\epsilon^{2}}-v_{1} \frac{\partial_{x} f_{0}(s)}{\epsilon^{2}}\right] d s .
\end{aligned}
$$

Approximating the integral by an integral over step functions defined in each interval of length $\Delta t$ and approximating the derivative with respect to $x$ as before, we get for $t-n \Delta t$

$$
\begin{aligned}
\ddot{f}_{0}(t)= & e^{-\frac{t \sigma}{\epsilon^{2}} g(x)+} \\
& \sum_{k=0}^{n-1} \frac{\epsilon^{2}}{\sigma} e^{-\frac{(n-k-1) \Delta t \sigma}{\epsilon^{2}}}\left(1-e^{-\frac{\Delta t \sigma}{\epsilon^{2}}}\right)\left[\frac{\sigma K f_{0}^{(k)}}{\epsilon^{2}}-v_{1} \frac{D^{+}}{\Delta x} f_{1}^{(k)}+G\right] \\
\vec{f}_{1}(t)= & \sum_{k=0}^{n-1} \frac{\epsilon^{2}}{\sigma} e^{-\frac{(n-k-1) \Delta t-\pi}{\epsilon^{2}}}\left(1-e^{-\frac{\Delta t+2}{\epsilon^{2}}}\right)\left[\frac{\sigma K f_{1}^{(k)}}{\epsilon^{2}}-\frac{v_{1}}{\epsilon^{2}} \frac{D}{\Delta x} f_{0}^{(k)}\right]
\end{aligned}
$$

where we defined $f_{0}^{(k)}=f_{0}(k \Delta t), k=0, \ldots, n-1$. 
Reconsidering equations $(5.1,5.2)$ and $(5.3,5.4)$ for Step 1 and Step 2 of the numerical scheme and putting the steps together, we get for one time step

$$
\begin{array}{r}
\quad f_{0}^{k+1}=A f_{0}^{k}-A v_{1} \Delta t \frac{D^{+}}{\Delta x} f_{1}^{k}+A G \Delta t \\
+B<f_{0}^{k}>-B \Delta t \frac{D_{1}}{\Delta x}<v_{1} f_{1}^{k}>+B G \Delta t
\end{array}
$$

or

$$
\begin{aligned}
& f_{0}^{n}=A^{n} f_{0}^{0}+\sum_{k=0}^{n-1} A^{n-k-1} \\
& {\left[-A v_{1} \Delta t \frac{D^{+}}{\Delta x} f_{1}^{k}+A G \Delta t+B<f_{0}^{k}>-B \Delta t \frac{D^{+}}{\Delta x}<v_{1} f_{1}^{k}>+B G \Delta t\right]}
\end{aligned}
$$

or

$$
\begin{array}{r}
f_{0}^{n}=A^{n} g+\sum_{k=0}^{n-1} A^{n-k-1} B\left[-\frac{\epsilon^{2}}{\sigma} v_{1} \frac{D^{+}}{\Delta x} f_{1}^{k}+G \frac{\epsilon^{2}}{\sigma}+<f_{0}^{k}>\right] \\
+\Delta t \sum_{k=0}^{n-1} A^{n-k-1} B\left[-\frac{D^{+}}{\Delta x}<\eta_{1} f_{1}^{k}>+G\right]
\end{array}
$$

and a similar formula for $f_{1}^{n}$.

In the following we want to estimate the consistency error, i.e. the difference between the true solution $\left(f_{0}(t) ; f_{1}(t)\right)$ of the inlegral equation $(6.1,6.2)$ and the value $\left(\tilde{f}_{0}^{n}, \tilde{f}_{1}^{n}\right)$ that is obtained by introducing the true solution $\left(f_{0}^{(k)}, f_{1}^{(k)}\right)=\left(f_{0}(k \Delta t), f_{1}(k \Delta t)\right)$ instead of $\left(f_{0}^{k}, f_{1}^{k}\right), k=0, \ldots, n-1$ into the above formula $(6.3)$.

This means we estimate the difference between $\left(f_{0}(t), f_{1}(t)\right)$ and $\left(f_{0}^{n}, f_{1}^{n}\right)$ with

$$
\begin{array}{r}
f_{0}^{n}=A^{n} g+\sum_{k=0}^{n-1} A^{n-k-1} B\left[-\frac{\epsilon^{2}}{\sigma} v_{1} \frac{D^{+}}{\Delta x} f_{1}^{(k)}+G \frac{\epsilon^{2}}{\sigma}+<f_{0}^{(k)}>\right] \\
+\Delta t \sum_{k=0}^{n-1} A^{n-k-1} B\left[-\frac{D^{+}}{\Delta x}<v_{1} f_{1}^{(k)}>+\left({ }^{\prime}\right]\right.
\end{array}
$$

and a similar formula for $f_{1}^{n}$. Restricting in the proof for simplicity to the case $K=\left\langle>\right.$, i.e $A=\left(1+\frac{\Delta t}{\tau^{2}} \sigma\right)^{-1} I$, we concentrate in the following on proving a pointwise estimate for

$$
\left|f_{0}(t)-\tilde{f}_{0}^{\prime \prime}\right|
$$

The proof is based on four lemmas.

LEMMA 6.1.

$$
\left|f_{0}(t)-f_{0}(t)\right| \leq C(\Delta t+\Delta x)
$$

where $C$ is a constant independent of $\epsilon$.

Proof.

$$
\left|f_{0}(t)-f_{0}(t)\right|
$$




$$
\begin{aligned}
\leq & \sum_{k=0}^{n-1} \mid \int_{k \Delta t}^{(k+1) \Delta t} \frac{\sigma}{\epsilon^{2}} e^{-\frac{(t-s) \sigma}{\epsilon^{2}}}\left[<f_{0}>(s)-\frac{\epsilon^{2} v_{1}}{\sigma} \partial_{x} f_{1}(s)+\frac{\epsilon^{2} G}{\sigma}\right] d s \\
& -e^{-\frac{(n-k-1) \Delta t a}{\epsilon^{2}}}\left(1-e^{-\frac{\Delta t \sigma}{\epsilon^{2}}}\right)\left[<f_{0}^{(k)}>-\frac{\epsilon^{2} v_{1}}{\sigma} \frac{D^{+}}{\Delta x} f_{1}^{(k)}+\frac{\epsilon^{2} G}{\sigma}\right] \mid \\
\leq \quad & C \sum_{k=0}^{n-1}\left|\int_{k \Delta t}^{(k+1) \Delta t} \frac{\sigma}{\epsilon^{2}} e^{-\frac{(t-s) \sigma}{\epsilon^{2}}}(\Delta t+\Delta x) d s\right| \\
& +\sum_{k=0}^{n-1}\left|\left[<f_{0}^{(k)}>-\frac{\epsilon^{2} v_{1}}{\sigma} \frac{D^{+}}{\Delta x} f_{1}^{(k)}+\frac{\epsilon^{2} G}{\sigma}\right]\right| \\
& {\left[\int_{k \Delta t}^{(k+1) \Delta t} \frac{\sigma}{\epsilon^{2}} e^{-\frac{(t-s) \sigma}{\epsilon^{2}}} d s-e^{-\frac{(n-k-1) \Delta t \sigma}{\epsilon^{2}}}\left(1-e^{-\frac{\Delta l \sigma}{2}}\right)\right] }
\end{aligned}
$$

where

$$
\begin{aligned}
& \left|<f_{0}>(s)-\frac{\epsilon^{2} v_{1}}{\sigma} \partial_{x} f_{1}(s)+\frac{\epsilon^{2} G}{\sigma}-\left(<f_{0}^{(k)}>-\frac{\epsilon^{2} v_{1}}{\sigma} \frac{D^{+}}{\Delta x} f_{1}^{(k)}+\frac{\epsilon^{2} G}{\sigma}\right)\right| \\
& \leq C(\Delta t+\Delta x)
\end{aligned}
$$

for $s \in[k \Delta t ;(k+1) \Delta t], \epsilon \leq \epsilon_{0}$ has been used. Since the second term is 0 , this is smaller than

$$
\begin{aligned}
& C(\Delta t+\Delta x) \sum_{k=0}^{n-1} e^{-\frac{\left(n-\frac{k-1) \Delta t a}{c^{2}}\right.}{2}}\left(1-e^{-\frac{\Delta t \pi}{c^{2}}}\right) \\
\leq & C(\Delta t+\Delta x) \sum_{k=0}^{n-1}\left(e^{-\frac{\Delta t \sigma}{c^{2}}}\right)^{k}\left(1-e^{-\frac{\Delta t \pi}{c^{2}}}\right) \\
\leq & C(\Delta t+\Delta x) .
\end{aligned}
$$

[

LEMMA 6.2 .

$$
\left|\Delta t \sum_{k=0}^{n-1} A^{n-k-1} B\left[-\frac{D^{+}}{\Delta x}<v_{1} f_{1}^{(k)}>+G\right]\right| \leq C \Delta t
$$

where $C$ is a constant independent of $\epsilon$.

Proof.

$$
\begin{aligned}
& \quad\left|\Delta t \sum_{k=0}^{n-1} A^{n-k-1} B\left[-\frac{D^{+}}{\Delta x}<v_{1} f_{1}^{(k)}>+G\right]\right| \\
& \leq C \Delta t\left|B \sum_{k=0}^{n-1} A^{n-k-1}\right|
\end{aligned}
$$

with $|A|=\left|\left(1+\frac{\Delta t}{\epsilon^{2}} \sigma\right)^{-1} I\right|<1$. This is equal to

$$
\begin{aligned}
& \quad C \Delta t\left|B \sum_{k=0}^{n-1} A^{k}\right| \\
& \leq C \Delta t\left|B(I-A)^{-1}\right| \\
& =C \Delta t,
\end{aligned}
$$


since $B=I-4$. $\square$ The estimates to prove the next Lemma can be found in [7]

LEMNA 6.3.

$$
\left|e^{-\frac{k \Delta t \pi}{\varepsilon^{2}}} I-A^{k}\right| \leq C \min \left(1 ; \frac{\sigma^{2}}{\epsilon^{4}} \Delta t k \Delta t\right)\left(1+\frac{\Delta t}{\epsilon^{2}} \sigma\right)^{-k}
$$

with $k=0, \ldots n$. In particular for $k=n$ one has

$$
\left|c^{-\frac{\operatorname{ton}}{2}} I \cdot A^{n}\right| \leq C \Delta t
$$

Proof.

We have

$$
\left|e^{-\frac{b \Delta \omega}{2}}\right| \leq\left(1+\frac{\Delta t \sigma}{\epsilon^{2}}\right)^{-k},\left|A^{k}\right| \leq\left(1+\frac{\Delta t \sigma}{\epsilon^{2}}\right)^{-k}
$$

This gives

$$
\left|e^{-\frac{k \Delta \alpha}{c^{2}}} I-A^{k}\right| \leq 2\left(1+\frac{\Delta t}{\epsilon^{2}} \sigma\right)^{-k}
$$

Moreover, since

$$
\ln \left(1+\frac{\Delta t \sigma}{\epsilon^{2}}\right)=\frac{\Delta t \sigma}{\epsilon^{2}}+O\left(\left(\frac{\Delta t \sigma}{\epsilon^{2}}\right)^{2}\right)
$$

we get:

$$
\begin{aligned}
& \left|e^{-\frac{k+t g}{2} I-A^{k}}\right| \\
& =\left|e^{-\frac{k \Delta t d}{c^{2}}}\left(1-e^{\left[\frac{k \Delta t \pi}{e^{2}}-k \cdot \ln \left(1+\frac{\Delta l \pi}{\epsilon^{2}}\right)\right]}\right)\right| \\
& =\left|e^{-\frac{k \Delta t a}{c^{2}}}\left(1-e^{-k O\left(\frac{\Delta t a}{k^{2}}\right)^{2}}\right)\right| \\
& \leq e^{-\frac{\Delta \Delta t \sigma}{c^{2}}} k O\left(\frac{\Delta t \sigma}{\epsilon^{2}}\right)^{2} \\
& \leq \quad k O\left(\frac{\Delta t \sigma}{\epsilon^{2}}\right)^{2}\left(1+\frac{\Delta t \sigma}{\epsilon^{2}}\right)^{-k} .
\end{aligned}
$$

Estimates (6.4) and (6.5) give the first assertion. To prove the second assertion we use the first one with $k=n, t=n \Delta t$ :

$$
\left|e^{-\frac{t \pi}{\epsilon^{2}}} I-A^{n}\right| \leq C \min \left(1, \frac{\sigma^{2}}{\epsilon^{4}} t \Delta t\right)\left(1+\frac{\Delta t}{\epsilon^{2}} \sigma\right)^{-n}
$$

However, this is smaller than $C \Delta t$, since, if $\frac{\Delta t t}{\epsilon^{2}}>\frac{1}{2}$ we have that it is smaller than

$$
C\left(\frac{3}{2}\right)^{-n} \leq \frac{C}{n} \leq C \Delta t
$$

if $\frac{\Delta i q}{c^{2}}<\frac{1}{2}$ we get that it is smaller than

$$
\frac{C}{n}\left(n \sigma \frac{\Delta t}{\epsilon^{2}}\right)^{2}\left(1+\frac{\left(n \sigma \frac{\Delta t}{\epsilon^{2}}\right)}{n}\right)-n \leq \frac{C}{n} \leq C \Delta t
$$

( 
LEMMA 6.4 .

$$
\left|f_{0}(t)-f_{0}^{n}\right| \leq C \Delta t+C \min \left(\frac{\epsilon^{2}}{\Delta t}, \frac{\Delta t}{\epsilon^{2}}\right),
$$

where $C$ is a constant independent of $\epsilon$.

Proof:

$$
\begin{aligned}
& || \hat{f}_{0}(t)-\tilde{f}_{0}^{n} \mid \\
\leq & \left|\Delta t \sum_{k=0}^{n-1} A^{n-k-1} B\left[-\frac{D^{+}}{\Delta x}<v_{1} f_{1}^{(k)}>+G\right]\right| \\
+ & \left|\left(e^{-\frac{1 \sigma}{c^{2}}} I-A^{n}\right) g\right| \\
+ & \left|\sum_{k=0}^{n-1}\left(e^{-\frac{(n-k-1) \Delta t \sigma}{c^{2}}}\left(1-e^{-\frac{\Delta t \sigma}{\epsilon^{2}}}\right) T-A^{(n-k-1)} B\right)\left[<f_{0}^{(k)}>-\epsilon^{2} \frac{v_{1}}{\sigma} \frac{D^{+}}{\Delta x} f_{L}^{(k)}+\epsilon^{2} \frac{G}{\sigma}\right]\right| .
\end{aligned}
$$

The first two terms are estimated by Lemma 2 and Lemma 3. They are smaller than

$C \Delta t$.

The third term is for $\epsilon \leq \epsilon_{0}$ smaller than

$$
\begin{array}{r}
C\left|\sum_{k=0}^{n-1}\left(e^{-\frac{(n-k-1) \Delta t \sigma}{\epsilon^{2}}}\left(1-e^{-\frac{\Delta t \sigma}{\epsilon^{2}}}\right) I-A^{(n-k-1)} B\right)\right| \\
\leq C\left|\sum_{k=0}^{n-1}\left(e^{-\frac{(n-k-1) \Delta t \sigma}{\epsilon^{2}}} I-A^{(n-k-1)}\right)\left(1-e^{-\frac{\Delta t \tau}{\epsilon^{2}}}\right)\right| \\
+C\left|\sum_{k=0}^{n-1}\left(\left(1-e^{-\frac{\Delta t \tau}{\epsilon^{2}}}\right) I-B\right) A^{(n-k-1)}\right|
\end{array}
$$

Using $I-B=A$ and Lemma 3 with $k=1$ the second term on the right hand side in (6.6) is smaller than

$$
\begin{aligned}
& C \sum_{k=0}^{n-1}\left|A^{k}\right|\left|\left(1-e^{-\frac{\Delta t o}{\epsilon^{2}}}\right) I-B\right| \\
\leq & C\left|(I-A)^{-1}\right|\left|A-e^{-\frac{\Delta t \sigma}{c^{2}}} I\right| \leq C\left(1+\frac{\Delta t \sigma}{\epsilon^{2}}\right)\left(\frac{\Delta t \sigma}{\epsilon^{2}}\right)^{-1} \min \left(1,\left(\frac{\Delta t \sigma}{\epsilon^{2}}\right)^{2}\right)\left(1+\frac{\Delta t \sigma}{\epsilon^{2}}\right)^{-1} \\
= & C\left(\frac{\Delta t \sigma}{\epsilon^{2}}\right)^{-1} \min \left(1,\left(\frac{\Delta t \sigma}{\epsilon^{2}}\right)^{2}\right) \leq C \min \left(1,\left(\frac{\Delta t \sigma}{\epsilon^{2}}\right)^{2}\right) \frac{\epsilon^{2}}{\Delta t \sigma} \\
\leq & C \min \left(\frac{\Delta t}{\epsilon^{2}}, \frac{\epsilon^{2}}{\Delta t}\right)
\end{aligned}
$$

Again due to Lemma 3 with $k$ substituted by $n-k-1$ the first term in $(6.6)$ is smaller than

$$
\begin{array}{r}
C\left(1-e^{-\frac{\Delta t \sigma}{\epsilon^{2}}}\right) \sum_{k=0}^{n-1} \min \left(1,\left(\frac{\Delta t \sigma}{\epsilon^{2}}\right)^{2}(n-k-1)\right)\left(1+\frac{\Delta t \sigma}{\epsilon^{2}}\right)^{-(n-k-1)} \\
\leq C \min \left(1, \frac{\Delta t \sigma}{\epsilon^{2}}\right) \sum_{k=0}^{n-1} \min \left(1, k\left(\frac{\Delta t \sigma}{\epsilon^{2}}\right)^{2}\right)\left(1+\frac{\Delta t \sigma}{\epsilon^{2}}\right)^{-k}
\end{array}
$$


since $1-e^{-x} \leq \min (1, x)$. For $\frac{\Delta t \sigma}{\epsilon^{2}}>1$ this is smaller than

$$
\leq C \sum_{k=1}^{n-1}\left(1+\frac{\Delta t \sigma}{\epsilon^{2}}\right)^{-k} \leq C \frac{\epsilon^{2}}{\Delta t \sigma}
$$

For $\frac{\Delta t \sigma}{\epsilon^{2}}<1$ it is smaller than

$$
\begin{aligned}
& C \frac{\Delta t \sigma}{\epsilon^{2}} \sum_{k=0}^{n}\left(\frac{\Delta t \sigma}{\epsilon^{2}}\right)^{2} k\left(1+\frac{\Delta t \sigma}{\epsilon^{2}}\right)^{-k} \leq C\left(\frac{\Delta t \sigma}{\epsilon^{2}}\right)^{3} \sum_{k=0}^{n-1} k\left(1+\frac{\Delta t \sigma}{\epsilon^{2}}\right)^{-k} \\
& \leq C\left(\frac{\Delta t \sigma}{\epsilon^{2}}\right)^{3} \frac{\left(1+\frac{\Delta t \sigma}{\epsilon^{2}}\right)^{-1}}{\left(1-\left(1+\frac{\Delta t \sigma}{\epsilon^{2}}\right)^{-1}\right)^{2}}=C \frac{\Delta t \sigma}{\epsilon^{2}}\left(1+\frac{\Delta t \sigma}{t^{2}}\right) \leq C \frac{\Delta t \sigma}{\epsilon^{2}} 2
\end{aligned}
$$

due to $\sum_{k=0}^{\infty} k x^{k}-\frac{x}{(1-x)^{2}},|x|<1$. This means that the first term in $(6.6)$ is smaller than

$$
\operatorname{Cmin}\left(\frac{\Delta t}{\epsilon^{2}}, \frac{\epsilon^{2}}{\Delta t}\right)
$$

with $C$ independent of $\epsilon$. Collecting all the terms the lemma is proven. $\square$ All together, using Lemma 1 and 4 we have proven for $t \in[0, T], t=n \Delta t$

$$
\left|f_{0}(t)-\tilde{f}_{0}^{n}\right| \leq\left|f_{0}(t)-\bar{f}_{0}(t)\right|+\left|f_{0}(t)-f_{0}^{n}\right| \leq C(\Delta t+\Delta x)+C \min \left(\frac{\epsilon^{2}}{\Delta t}, \frac{\Delta t}{\epsilon^{2}}\right) .
$$

This means, that for small $\Delta t, \Delta x$ and fixed $t$, the estimate tends to 0 like $\Delta t+\Delta x$.

However, also for a meshsize, that is large compared to $\epsilon$ the estimate shows, that we get convergence to 0 . For example, for $c \leq C \Delta t$ we obtain convergence to 0 like $\Delta t+\Delta x$.

We mention that $\Delta t$ has to be chosen in relation to $\Delta x$. E.g. in the diffusion limit we need $\Delta t$ to be of the order of $(\Delta x)^{2}$ as we have seen in the last section.

7. Numerical Results and Examples. In this section a numerical study of the scheme is presented and the scheme is compared with fully explicit and fully implicit schemes.

We restrict to the one-group transport equation in slab geometry, i.e. $x \in[0, L]$ and $K=<>$. This yields $D=\frac{1}{3}$. The velocity discretization is done using in all situations a 16 point quadrature set.

We compure the solution with the semi-implicit schene derived above for different space discretizations. To obtain positivity and stability of the semi-implicit scheme in the limit $e$ tending to 0 one has to take - for a fixed space discretization $\Delta x-a$ time step $\Delta t$ of the size given by (5.6). As mentioned above this can be relaxed for large $\epsilon$. In particular; this means that the size of $\Delta t$ can be chosen independent of $\epsilon$.

Comparison with the explicit scheme (3.1):

In contrast to the above we get that the explicit discretization (3.1) of equation (2.2) requires a time step of the order $\min \left(\Delta x \epsilon, \frac{\epsilon^{2}}{\sigma}\right)$ to obtain positivity and stability. In particular, for small $\epsilon$ the step size $\Delta t$ has to be chosen in this case of the order $\epsilon^{2}$, in contrast to the semi-implicit scheme. A comparison of the CPU time necessary for one time step yields that the semi-implicit scheme needs about 2 times the CPU time of the explicit scheme. This yields a big gain in computing time for small $\epsilon$ for the semi-implicit scheme compared to an explicit one. In particular, it is reasonable to use 
the semi-implicit scheme, if $2 \cdot \min \left(\Delta x \epsilon, \frac{\epsilon^{2}}{\sigma}\right)$ is smaller than $3 \frac{(\Delta x)^{2}}{2} \sigma$ and if the desired accuracy does not require a smaller time step, than the one that can be taken for the semi-implicit scheme. To obtain a certain required accuracy of the solution one has to use lime sleps as shown in the table below for some examples, see Table 1. Looking at Table 2 one observes that using an explicit scheme is not reasonable for small $\epsilon$. Either the semi-implicit or the implicit scheme are faster. However; this changes for $\epsilon$ large, where the explicit scheme may be better due to the small computation times per time step.

Comparison with the fully implicit scheme (3.2):

A fully implicit dicretization of the equation obviously allows bigger time steps, since there is no stability restriction on the time step in this case. Nevertheless, for the accurate simulation of the time development small time steps may be necessary. To get an accurate resolution of the behaviour of the solution up to an error of a certain order the size of the time step for the implicit scheme has to be chosen according to Table 1 below.

An implementation of a fully implicit scheme shows that in order to obtain a sufficient accuracy the stationary equation has to be evaluated to a very high accuracy. approximately up to an error of the order $10^{-8}$. To achieve this a standard iteration scheme using for example a diamond difference discretization needs a large number of iteration steps (sweeps over the computational domain). A comparison of the CPU: time for one iteration step shows that one time step of the semi-implicit iteration needs less than 2 times the CPU-time of an iteration of the stationary scheme. Table 2 shows that the semi-implicit scheme has a big advantage compared to a standard implicit iteration in many situations.

However, of course, computation times for an implicit scheme are strongly reduced if a multigrid algorithms as described, e.g., in [24] is used. Using the convergence estimates in [24] one observes that in essentially two $V(1,1)$ cycles an accuracy of the one needed for the solution of the stationary equation is obtained. One $V(1,1)$ cycle costs about the same CPU time as 4 sweeps over the computational domain. I.e. the estimated costs for one time step of a fully implicit scheme with a multigrid algorithm: is about 4 times as large as the one for the semi-implicit schome. The complexity of the implementation of a multigrid scheme especially in higher dimensions has to be taken into consideration as well.

We consider a situation with $\sigma=1, \sigma_{A}=0, G=0, \epsilon \leq 0.01$ and boundary conditions equal to 0 at $x=0$ and equal to 1 at $x=1$. The space discretizations are $\Delta x=0.1, \Delta x=0.01$. The time steps required for the semi-implicit scheme by stability considerations are in this case $\Delta t=0.015, \Delta t=0.00015$. We consider final times $t=0.005,0.05$ and 0.5 . For $t=0.5$ a stationary state is nearly reached. The error was calculated by taking the $\mathcal{L}^{1}$-norm of the difference with the 'true' solution computed with a very fine discretization. The table shows the time steps necessary. to obtain a certain accuracy $e$ with the implicit scheme using a diamond difference discretization.

\begin{tabular}{|c|c|c|}
\hline & $\Delta x=0.1, e: 5 \cdot 10^{-3}$ & $\Delta x=0.01, e: 5 \cdot 10^{-4}$ \\
\hline $\mathrm{t}=0.005$ & $*$ & $\Delta t=0.00015$ \\
\hline $\mathrm{t}=0.05$ & $\Delta t=0.003$ & $\Delta t=0.001$ \\
\hline $\mathrm{t}=0.5$ & $\Delta t=0.01$ & $\Delta t=0.004$ \\
\hline
\end{tabular}

Table 1: Time steps required to obtain a certain accuracy $e$.

Ihese accuracy requirements together with the above estimated CPU time give the following relation between the CPU time for the explicit (E), the semi-implicit (S) 


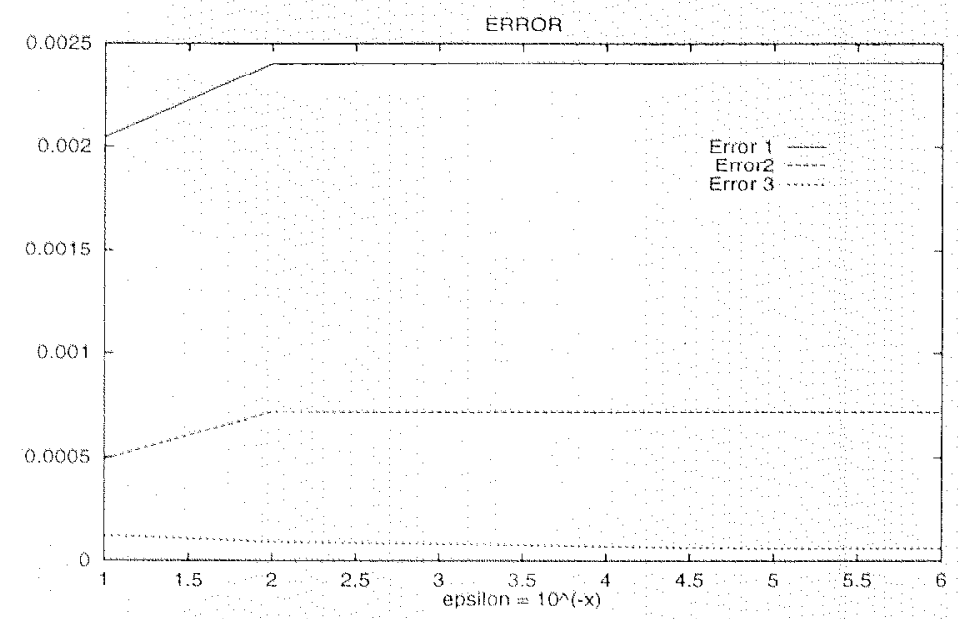

FIG. 7.1 Error for different values of $\mathrm{a}$

and the implicit scheme with multigrid (IM) and with a standard iteration procedure (IS) for $\epsilon \leq 0.01$ :

\begin{tabular}{|c|c|c|c|c|c|c|c|c|}
\hline & \multicolumn{3}{|c|}{$\Delta x=0.1, e \cdot 5 \cdot 10^{-3}$} & \multicolumn{3}{|c|}{$\Delta x=0.01, e \cdot 5 \cdot 10^{-1}$} \\
\hline & $\mathrm{E}$ & $\mathrm{S}$ & $\mathrm{IM}$ & $\mathrm{IS}$ & $\mathrm{E}$ & $\mathrm{S}$ & $\mathrm{IM}$ & $\mathrm{IS}$ \\
\hline $\mathrm{t}=0.005$ & $*$ & $*$ & $*$ & $*$ & $0.75 \cdot 10^{-1} \epsilon^{-2}$ & 1 & 4 & 15 \\
\hline $\mathrm{t}=0.05$ & $1.5 \cdot 10^{-3} \epsilon^{-2}$ & 1 & 4 & 220 & $0.75 \cdot 10^{-1} \epsilon^{-2}$ & 1 & $4 \cdot 0.15$ & 13 \\
\hline $\mathrm{t}=0.5$ & $0.5 \cdot 10^{-2} \epsilon^{-2}$ & 1 & 4 & 320 & $0.75 \cdot 10^{-1} \epsilon^{-2}$ & 1 & $4 \cdot 0.0375$ & 9 \\
\hline
\end{tabular}

Table 2: Relative CPU times

This shows that for coarse grids the semi-implicit scheme has to be prefered. For finer grids and nearly stationary situations the advantage of a fully implicit scheme with multigrid is clearly seen. Implicit schemes with a standard iteration procedure are in all considered situations slower than the semi-implicit scheme.

Further investigation of the semi-implicit scheme:

To show the uniform convergence in 6 for the semi-implicit schene numerically, we compute the error for different values of $\epsilon$ ranging from $\epsilon=0.1$ up to $\epsilon=10^{-6}$. As before, we use $\sigma=1, \sigma_{A}=0, G=0$, boundary conditions cqual to 0 and 1 at $x=0$ and $x=1$, respectively, and the following values for the space discretization $\Delta x$ with the corresponding $\Delta t$ values due to the stability condition $(5.6)$ :

$$
\Delta x=0.1,0.05,0.025,0.0125 \text {. }
$$

Hence, each cell contains between 0.125 and $10^{5}$ mean free paths. The error was calculated by taking the $L^{1}$-norm of the difference of the solutions with discretization size 0.0125 and 0.025 (error 3 ), 0.025 and 0.05 (error 2 ), 0.05 and 0.1 (error 1 ) respectively. This results in three curves, which are plotted in Figure 7.1. Looking at the figure, one observes that the error behaves perfectly uniform as $\epsilon \rightarrow 0$.

The solution of the kinetic equation computed by the new scheme derived in this work is in the following computed for different physical situations. The physical examples under consideration are

Example 1: $\epsilon=1, \sigma=1, \sigma A=0, G(x)=0, L=1$, the boundary conditions $f(0, v)=0, v_{1}>0, f(L, v)=1, v_{1}<0$ and $t=4$.

Example 2: $\epsilon=0.01, \sigma=1, \sigma_{A}=0, G(x)=1.0, L=1$, the boundary conditions are equal to 0 and $t=0.4$. 


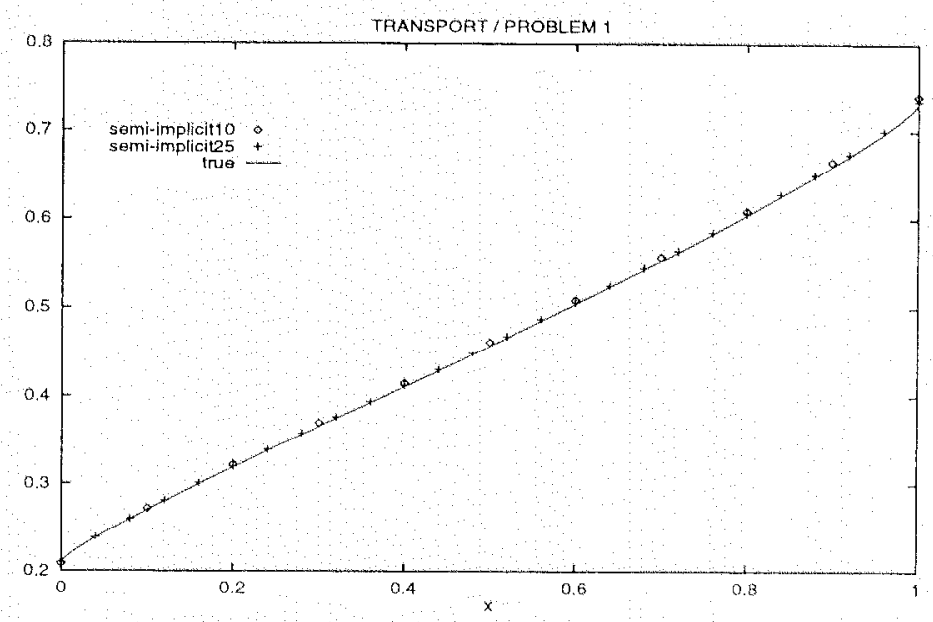

FIG. $7.2 . \epsilon=1, t=4, G=0, \sigma=1$

Example 3: $\epsilon=0.001, G(x)=1.0, I=1$, boundary conditions equal to $0, \sigma(x)=$ $1+x, \sigma_{A}=0$ and $t=0.4$.

Example 4: $\epsilon=0.01, \sigma=1, \sigma_{A}=0, G(x)=0.0, L=1$ and boundary conditons $f(0, v, t)=v_{1}, v_{1}>0, f(L, v, t)=0, v_{1}<0$ and $t=0.4$. The solution of this problem has a kinetic boundary layer at $x=0$.

Example 5: As Example 4, but with $\epsilon=0.0001$.

Example 6: A two material problem. $L=1.1, t=1$. In $[0,0.1]$ we consider a purely absorbing material with $\sigma=0, \sigma_{A}=1, G(x)=0$ and $\epsilon=0.1$. I.e. the region has the size of one mean free path. In $[0.1,1.1]$ we take a purely scattering material $\sigma=1, \sigma_{A}=0, G(x)=0$ and $\epsilon=0.001$ (1000 mean free paths in this region). The boundary conditions are $f(0, v)=1, v_{1}>0, f(L, v)=0, v_{1}<0$. The solution of this problem has an interface layer at $x=0.1$.

The initial condition is always 0 .

The solutions for the physical situations described above are plotted in the following figures. In Figure 7.2 to 7.4 the situations from Example 1 to 3 are shown. The solutions are plotted using space discretizations $\Delta x-0.04$ and $\Delta x-0.1$ for the semi-implicit scheme. We use the label 'semi-implicit10' to denote the solution with the semi-implicit scheme with 10 spatial cells. The time discretization is choscn duc to the stability condition (5.6) for Example 2 and 3. For Example 1 the restriction on the time step is relaxed to a CFI mtype condition. The reference solution is the solution with a very fine discretization. For this case the solution of the semi-implicit scheme and of the other schemes are coincident. The solution of the diffusion equation is computed by the usual triangular explicit scheme, which is the limiting scheme of our semi-implicit scheme as $\epsilon$ tends to 0 , compare (4.3). The example shows that for isotropic boundary conditions the solution is approximated with good accuracy for different ranges of $\epsilon$.

In Figure figure layer 1 Example 4 is considered. We plot the reference solution and the solution of the diffusion equation with boundary coefficients derived from the halfspace problem. The solutions of the semi-implicit scheme are found with $\Delta x=0.1$ such that a discretization cell contains 10 mean free paths and the corresponding size of the time discretizalion. The boundary values are found by determining approximately the outgoing distribution of the halfspace problem (2.3) as described in Section 3. 


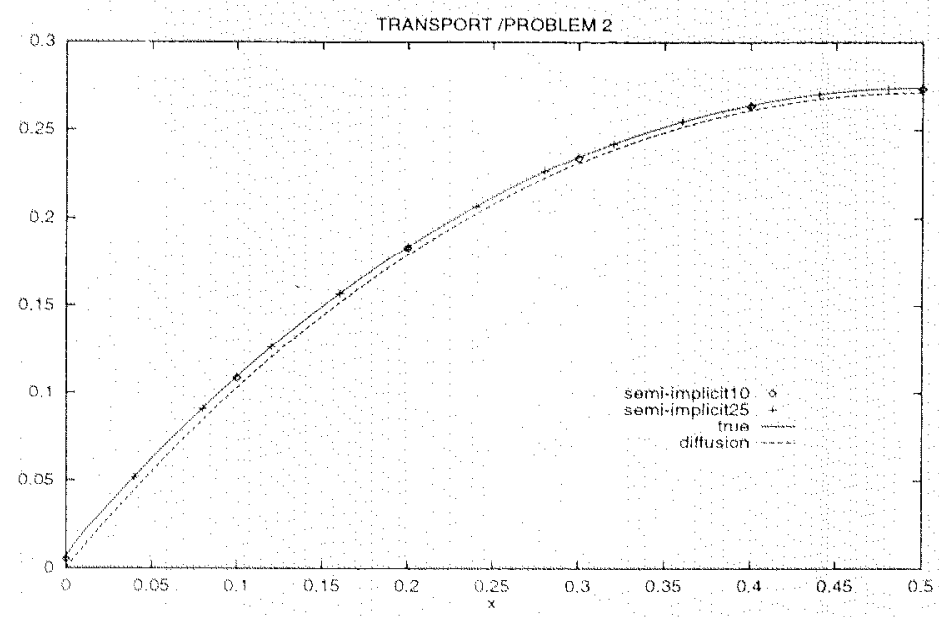

FIG, $7.3 .6=0.01, t=0.4, G=1.0, \sigma=1$

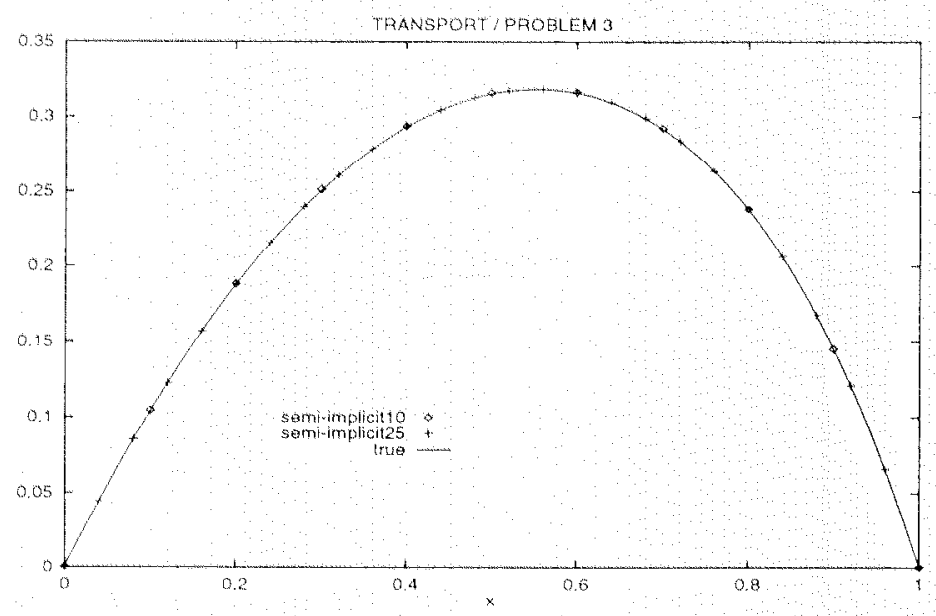

FIG. $7,1, t=0.001, t=0.4, G=1, \sigma=1+x$

This is done using first the approximation of the asymptotic value of the halfspace problem by (3.7), as the outgoing function (the solution in the plot is labeled semiimplicit10-1) and second an outgoing function determined by formula (3.10) labeled 'semi-implicit 10-2'. In this first case the two approaches give comcident results. One observes that even for a coarse diffusive discretization the behavion of the solution at the boundary is found with very good accuracy. We mention that other approaches to obtain the correct discrete boundary conditions for the stationary equation are shown in $[14,21]$.

Figure 7.6 shows Example 5. The same as in Figure 7.5 is shown. However, in this case one cell contains now 1000 mean free paths. The advantage of using here an exact approximation of the outgoing fiunction of the half space problem is clearly seen.

Finally Figure 7.7 shows Example 6. The space discretization is here $\Delta x=0.005$ in the absorbing region and $\Delta x=0.1$ in the scattering region. In particular, one cell in the scattering region contains 100 mean free paths. The situation at the interface 


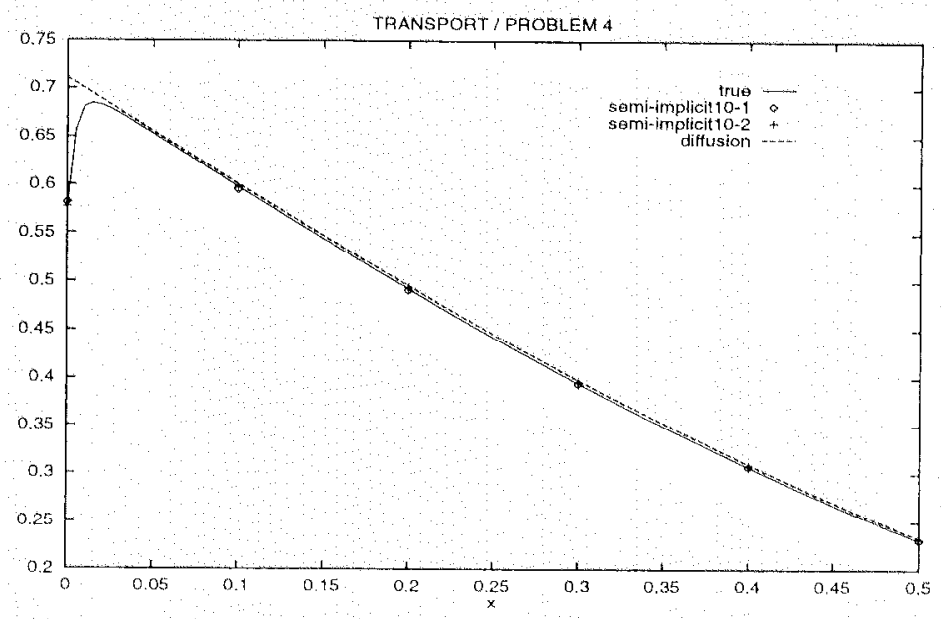

FIG. $7.5 . \epsilon=0.01, t=0.4, G=0, \sigma=1$

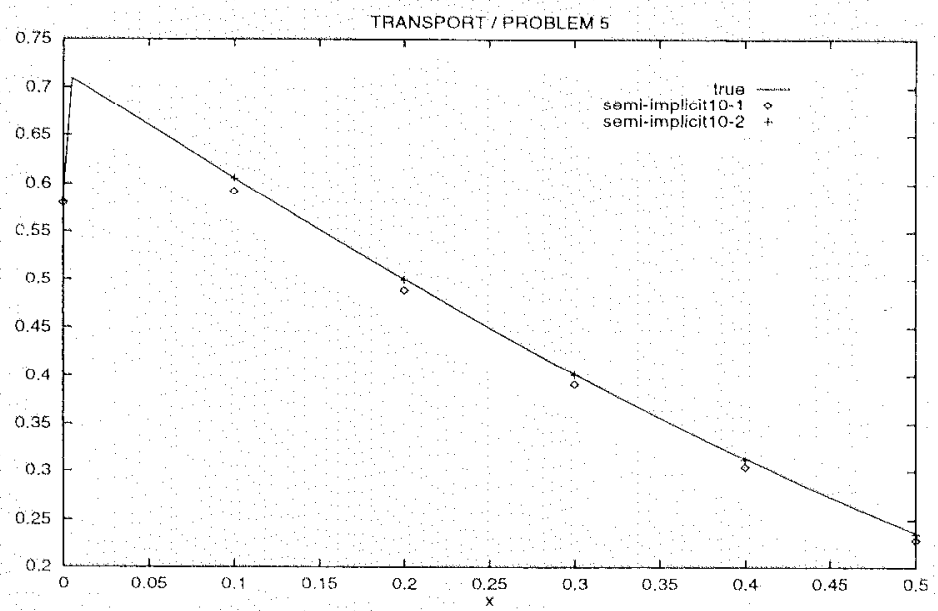

FIG. 7.6. $t=0.0001, t=0.4, G=0, \sigma=1$

is treated in the same way as the one at the boundaries before. One observes again a good agreement of the solution in the diffusive region with the true solution. We mention here the work of $[14,18,21]$ who treated similar problems for the stationary equation. 


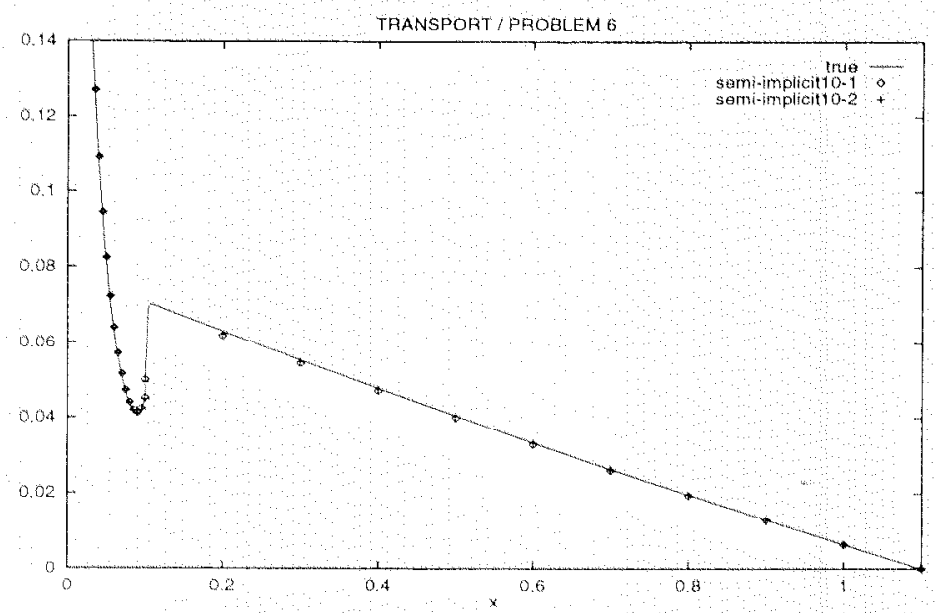

Fia. 7.7. $\epsilon=0.1 / 0.001, t=1, G=0, \sigma=0 / 1,0 A=1 / 0$

8. Conclusions. From the analytical and numcrical results onc can conclude:

- The semi-implicit scheme works uniformly for all ranges of the mean free path. This is shown by numerical experiments and a consistency proof.

- The limiting schene for small mean free paths is a standard explicit discretization of the diffusion equation.

- By including a boundary layer analysis one obtains a suitable treatment of the boundary conditions for coarse (diffusive) discretizations.

- A comparison of the scheme with fully explicit and fully implicit schemes shows advantages and disadvantages. In particular, the semi-implicit scheme is faster than the fully implicit scheme, if the detailed time development is computed with a coarse discretization or with higher accuracy reculirements. However, for nearly stationary situations with a fine grid the fully implicit scheme, if combined with a fast multigrid method as in [24], is faster.

- The numerical results have been generated for the one group transport case. A further numerical treatment should include the implementation of the scheme with other scattering ratios. Using methods as in $[1,13]$ this should be possible without too much difficulties.

\section{REFERENCES}

[1]. K. ATKinson, A Survey of Numerical Methods for the solution of Fredholm Integral Equations. of the Second Kind, Society for industrial and applied mathematics, Philadelphia, 1976.

[2] C Bandos, F. Golse, and D. Levermore, Fuid dynamic limits of kinetic equations formal derivations, J. Stat. Phys, 63 (1991), p. 323.

[3] C. BARDOS, R. SANTOS, AND R SENTIS Diffusion approtimation and computation of the critical size, TAMS, 284 (1984), p. 617 .

[4] A. Bengoussan, I. LIONS, AND G. PAPANTOLAOU, Boundary layers and homogenzation of transport processes, Tubl. RIMS Kyoto Univ, 15 (1979), 15. 52 .

[5] I. BOBYLEV AND J. STRUCKMEIER, Implicit and iterative methods for the Boltzmann equation, TTSP, 25 (1996), P. 175

[6] R. CAFLISF, the fluid dynamical limit of the nonlinear Boltzmann equation, $\mathrm{CPAM}, 33$ (1980), p. 651 .

[7] R. CAFLISH, S.JIN, AND G. Russo, Uniformly accurate schemes for hyperbolic systems with relaxation, to appear in SIAM J, Num. Anal.

[8] C. Cercionani, The Boltzmann Equation and its Applications, Springer, 1988. 
[9] F. CORON AND B. PERTHAMe, Numerical passage from kinetic to fluid equations, SIAM J. Numer. Anal., 28 (1991), p. 26.

[1.0] A. DE MASI, R. Esposito, AND J. LEBOWIT, Incompressible Navier Stokes and Euler limits of the Boltzmann equation, CPAM, 42 (1989), p. 1189.

[11] F. GOLSE AND A. KLAR, A numerical method for computing asymptotic states ard outgoing distributions for kinetic linear half space problems, J. Stat. Phys., 80 (1995), p. 1033.

[12] G. HABETLER AND B. MATKOWSKY, Uniform asymptotic expansions in transport theory with small mean free paths, and the diffusion approximation, J. Math. Phys., 16 (1975), p. 846.

[13] W. HackBusch, Integralgleichungen: Theorie und Numerik, LAMM 68, Teubner, 1989, 1989.

[11] S. JIN AND D. LeVermone, Fully-discrete numerical transfer in diffusive regimes, TTSP, 22 (1993), p. 739.

[15] - Numerical schemes for hyperbolic conservation lawes with stiff relaxation terms; $\mathrm{J}$. Comp. Phys., 126 (1996), p. 449.

[16] S. JIN AND Z. XIN, The relatation schemes for systems of conservation laws in arbitrary space dimensions, Comm. Pure Appl: Mathi, 48 (1995), p. 235.

[17] A. KLAR, Asymptotic-induced domain decomposition methods for kinetic and drift diffusion semiconductor equations, to appear in SIAM J. Sci. Comp.

[18] E. LARSEN, Diffusion theory as an asymptotic limit of transport theory for nearly critical systems with small mean free paths, Ann. Nucl. Energy, 7 (1980), p. 249.

[19] — Transport acceleration methods as two level multigrid algorithms, Oper. Theory Adv. Appl., 51 (1991), p. 34.

[20] E. LARSEN AND J. KELLER, Asymptotic solution of neutron transport problems for small mean free path, J. Math. Phys., 15 (1974), p. 75.

[21] E. LARSEN AND J. MOREL, A symptotic solution of numerical transport problems in optically thick, diffusive regimes ii, J. Comp. Phys., 83 (1989), p. 212.

[22] E. LARSEN, J. MOREL, AND W. MILLER, Asymptotic solution of numcrical transport problems in optically thick, diffusive regimes, J. Comp. Phys., 69 (1987), p. 283.

[23] E. Lewis and W. Miller, Computational Methods of Neutron Transport, John Wiley and Sons, 1984.

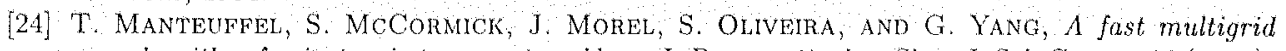
algorithm for isotropic transport problems I: Pure scattering, Siam J. Sci Comp., 16 (1995), p. 601 .

[25] T. MAN'EUfFe, S. MOCORMICK, J. MOREL, AND G. YANG, A fast multigrid algorithm for isotropic transport problems II: With absorption, Siam J. Sci. Comp., 17 (1996), p. 1449.

[26] T. MANTEUFFel AND K. Ressel, Multilevel methods for transport equations in diffusive regimes, in Proceedings of the Copper Mountain Conference on Multigrid Methods, April $5-9,1993$.

[27] G. MARCHUK, Methods of Numerical Mathematics, Springer, 1982.

[28] F. POUPAUD, Diffusion approximation of the linear semiconductor equation, J. Asympt. Anal., 4 (1991); p. 293. 